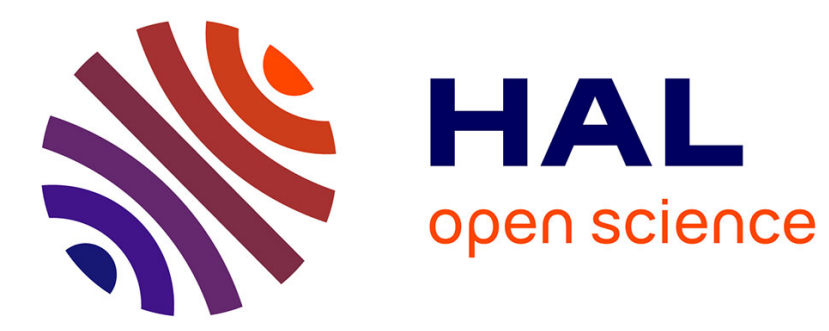

\title{
Capillary-size flow of human blood plasma: Revealing hidden elasticity and scale dependence
}

Ursula Windberger, Patrick Baroni, Laurence Noirez

\section{To cite this version:}

Ursula Windberger, Patrick Baroni, Laurence Noirez. Capillary-size flow of human blood plasma: Revealing hidden elasticity and scale dependence. Journal of Biomedical Materials Research Part A, 2021, 2021, pp.1-6. 10.1002/jbm.a.37286 . hal-03296449

\section{HAL Id: hal-03296449 \\ https://hal.science/hal-03296449}

Submitted on 22 Jul 2021

HAL is a multi-disciplinary open access archive for the deposit and dissemination of scientific research documents, whether they are published or not. The documents may come from teaching and research institutions in France or abroad, or from public or private research centers.
L'archive ouverte pluridisciplinaire HAL, est destinée au dépôt et à la diffusion de documents scientifiques de niveau recherche, publiés ou non, émanant des établissements d'enseignement et de recherche français ou étrangers, des laboratoires publics ou privés. 


\title{
Capillary-size Flow of Human Blood Plasma: Revealing Hidden Elasticity and Scale Dependence
}

\author{
U. Windberger ${ }^{1}$, P. Baroni², L. Noirez ${ }^{2}$ \\ ${ }^{1}$ Center for Biomedical Research, Decentralized Biomedical Facilities, Medical University Vienna, Borschkegasse 8a, \\ 1090 Vienna, Austria. ORCID: 0000-0002-5800-9089 \\ ${ }^{2}$ Laboratoire Léon Brillouin (CEA-CNRS), Univ. Paris-Saclay, CEA-Saclay, 91191 Gif-sur-Yvette, France. ORCID :
} 0000-0002-0164-1155

The dynamical mechanical analysis of blood generally uses models inspired by conventional flows, assuming scaleindependent homogeneous flows and without considering fluid-surface boundary interactions. The present experimental study highlights the relevance of using an approach in line with physiological reality providing a strong interaction between the fluid and the boundary interface. New dynamic properties of human blood plasma are found: a finite shear elastic response (solid-like property) is identified in nearly static conditions, which also depends on the scale (being reinforced at small scales). The elastic behavior is confirmed by the induction of local hot and cold thermodynamic states evidencing a thermo-mechanical coupling in blood plasma so far known only in elastic materials. This finding opens new routes for medical diagnosis and device fabrication.

Keywords: Blood plasma, Interactive surface, Shear-elasticity, Flow

Introduction

The transport mechanisms of blood in the vasculature face multiple biological, physical, and biochemical parameters that make their flow laws complex, specific, and fascinating. Ninety years after the first works from Fahraeus and Lindqvist ${ }^{1}$ showing the decrease of blood viscosity with decreasing tube diameter, characterizing physiological behavior remains a challenge. Blood behavior is not only influenced by the flow properties of blood cells, but also by their ability to couple with the surrounding plasma. Plasma is a colloidal aqueous solution having almost 10 mass $\%$ of proteins, smaller molecules, and ions $^{2}$. It plays a key role by interacting and dragging the blood cells in the vessels. While it was long time believed to flow 
uncoupled (like Newtonian liquids), there is more and more evidence for non-Newtonian plasma behavior ${ }^{3,4}$. The flow involves the displacement of fluid layers against each other, and therefore the breaking of the fluid cohesion. This intrinsic property is rarely discussed and measured. However, recent careful tests have shown that it is possible to access the fluid cohesion at small scale probing the stress response of the fluid upon applying a mechanical shear strain. It is shown that "simple" fluids as liquid water exhibit a resistance to shear flow at small scale. The liquid elasticity vanishes by increasing the scale, giving rise to the conventional viscous behavior ${ }^{5-8}$. The very early experimental evidence of liquid elasticity dates back to Derjaguin (in the $1980^{\prime}$ ) at few microns scales ${ }^{9}$. At macroscopic scale, rheology has systematized the viscoelastic study of fluids with well-established protocols based on the Maxwell model established by Frenkel in "The Kinetic Theory of Liquids" $(1946)^{10}$. Derjaguin's works were forgotten until new measurements obstinately pointed out that the surface/liquid boundary conditions impact the mesoscale viscoelastic response ${ }^{5-8}$. At the same time, theoreticians have taken up Frenkel's calculation and demonstrated that fluid viscoelastic moduli do not necessarily vanish at low frequency; an elastic approach is equally valid ${ }^{11-14}$. The liquid shear elasticity weakens as the inverse cubic power of confinement length $L$, being rather inaccessible at macroscopic scale ${ }^{12-14}$. Due to the high importance of the small scales in the blood circulation, identifying scale-dependent shear elasticity and possibly coupled thermo-elastic effects ${ }^{15}$ in human blood plasma is an utmost important challenge. To understand the underlying mechanisms and access the shear elasticity, we explored the mechanical and thermal plasma properties at different scales in relation with the microvascular dimensions.

We operated at optimized conditions to test the true fluid resistance to a dynamic mechanical shear strain, in a frequency range reproducing the heartbeat and at length scales compatible with physiological dimensions. We started from the observation that standard protocols used for viscometry or viscoelastic measurements cannot be satisfactory. Classical rheometry uses metallic surfaces that are unphysiological and the degree of "un-physiology" is not known. They do not reproduce the interactive role of endothelial lubrication that covers biological surfaces ${ }^{16,17}$. Classical rheometry protocol also does not consider the influence of the scale and excludes the diameters within the microvasculature. To approach true fluid properties it is necessary to adapt the dynamic mechanical measurement. We thus combine innovative conditions associating tribology and multi-scale mechanical dynamical analysis together with a simultaneous time-resolved thermo-mapping of the fluid upon strain field.

The methodology includes an optimal stress transfer through the liquid by enhancing the force of interaction of the fluid to the wall. Excellent interfacial wetting is a prerequisite to strengthen the interaction. The degree of wetting can be characterized by the method of the contact angle $\theta$ (Scheme 1). Slippage propensity is reduced when the contact angle is reduced $^{18}$. The improved wetting method showed already that ordinary liquids (classified Newtonian by classical rheometry) 
exhibited a solid-like response below an elastic threshold ${ }^{5,9,19,20}$. We felt therefore confident to find such elasticity also in blood plasma after removal of blood cells that usually provide the elasticity ${ }^{21}$.
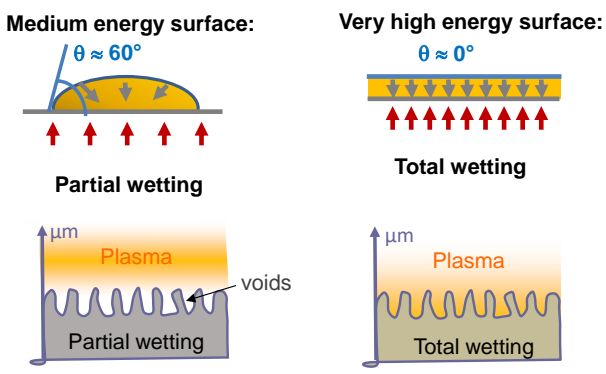

Scheme 1. Differences at macroscopic scale (upper schemes representing the different drop behaviors) and micron-scale (bottom schemes) between partial wetting (scheme showing an about $60^{\circ}$ contact angle) (a) and total wetting reaching zero contact angle (b) mechanisms. High-energy surfaces are required to provide surface boundary conditions in line with physiological interacting fluids.

\section{Materials and Methods}

We optimized the plasma - substrate interaction by means of zero-porosity $\alpha$-alumina surfaces. $\alpha$-alumina is chemically and biologically inert. Due to its electronic and atomic structure the $\alpha$-alumina is a high-energy surface ${ }^{22,23}$. Blood plasma contains $90 \%$ water fulfilling the total wetting conditions (illustrated in Scheme 1). The fluid was set in the gap at ambient temperature between two $\alpha$-alumina surfaces (plate-plate disks; $40 \mathrm{~mm}$ diameter). The gap thickness varied from $300 \mu \mathrm{m}$ to $1100 \mu \mathrm{m}$. The shear strain is defined as $\gamma=\delta l / e$, where $\delta l$ is the displacement and $e$ the gap thickness. The strain values range from $1 \%$ to $2000 \%$, depending on the gap thickness. A sin shear strain wave $\chi(\omega)$ was generated by the oscillatory motion of one surface while the other one is fixed and coupled to a stress sensor (using a strain imposed rheometer ARES2 (TAInstruments)). The resulting shear stress $\sigma(\omega)$ that measures the resistance of the fluid to the deformation is conventionally expressed in terms of macroscopic viscoelastic constants to characterize the dynamic fluid properties: $\sigma(\omega)=$ $\gamma_{0 .}\left(G^{\prime}(\omega) \cdot \sin (\omega \cdot t)+G^{\prime \prime}(\omega) \cdot \cos (\omega \cdot t)\right)$ where $\left(G^{\prime}\right)$ and $\left(G^{\prime \prime}\right)$ are the shear elastic and viscous moduli respectively ${ }^{24}$. These constants are by essence statistical quantities.

The temperature inside the fluid was simultaneously investigated using a micro bolometric detector array of $382 \times 288$ pixels embedded in a camera working at a frame rate of $27 \mathrm{~Hz}$. The camera integrates a lens with a focal length of $7.5 \mathrm{~mm}$ and a numerical aperture of $\mathrm{F} / \mathrm{d}=0.6$ detects the radiation emitted by the liquid surface in the LWIR band; i.e. wavelengths ranging between 7 and $14 \mu \mathrm{m}$. The thermal amplitude adjustment range is $\Delta \mathrm{T}=2^{\circ} \mathrm{C}$ and the thermal sensitivity $+/-0.02^{\circ} \mathrm{C}$. 
The front lens is placed $15 \mathrm{~mm}$ away from the liquid surface, ensuring non-contact measurements. The lens magnifies 20x. The depth of focus is defined by the pixel pitch $(20 \mu \mathrm{m})$, the lens aperture F/1 and, the distance between the object the lens pupil and the focal plane. The depth of thermal field is about $0.1 \mathrm{~mm}$. The images were corrected by subtraction of the equilibrium median value. The signals collected on several pixels are then added along each column (or the Y axis of the detector array). With this method, the Noise Equivalent Temperature Difference (NETD) is considerably reduced reaching typically values closed to $0.003 \mathrm{~K}$. After giving informed consent (2114/2019, MedUni Vienna, Austria), blood was obtained from one healthy volunteer after given informed consent (postmenopausal female, 55a, BMI: 24, non-smoker) by puncturing the antecubital vein with a $21 \mathrm{G}$ butterfly needle that was connected to a vacutainer system containing EDTA for anticoagulation. The study conforms to the Declaration of Helsinki. Whole blood was centrifuged to obtain platelet-depleted plasma (2310 $\mathrm{g}$ for 15 minutes, the upper two thirds of the plasma column was withdrawn and again centrifuged at $2310 \mathrm{~g}$ for 20 minutes). The superficial layer was discarded and the central fraction was collected and immediately essayed. Blood plasma was stored at room temperature in several containers and new portions were filled in the gap for each measurement. Finally, because of the high water content $(90 \%)$, blood plasma can be considered as a blackbody according to its transmission ${ }^{25,26}$ and is therefore perfectly adapted for an infrared study.

\section{Results}

Figure 1 displays the dynamic response of a $0.300 \mathrm{~mm}$ thickness blood plasma sample submitted to an oscillatory shear sine input over a wide frequency range $(0.04 \mathrm{rad} / \mathrm{s}-100 \mathrm{rad} / \mathrm{s})$. Low strain amplitude conditions were applied $(1.5 \%)$ to test the fluid in nearly equilibrium conditions. The elastic modulus $G^{\prime}$ dominated the dynamic response indicating that plasma responds nearly instantaneously to the oscillatory strain field within this frequency. Viscous dissipation was low, since $G^{\prime \prime}$ exhibited values three times lower than $G^{\prime}$. This means that plasma did not flow but oscillated as a whole in phase with the shear excitation. Both moduli increased at higher frequency, indicating a reinforcement of the strength at faster motions.

Second, the response of blood plasma was examined as a function of the strain amplitude at different thicknesses. Figure 2 displays the evolution of the shear elasticity as a function of the strain amplitude at small scale $(0.300 \mathrm{~mm})$ with the corresponding thermal mapping displayed at three shear strain amplitudes. Three dynamic regimes are distinguished. At relatively low strain amplitude $(\gamma<200 \%)$, the shear elastic modulus $G^{\prime}(\omega)$ was higher than the shear viscous modulus $G^{\prime \prime}(\omega)$. At higher strain amplitudes $(300 \%<\gamma<500 \%)$, both moduli collapsed with a more pronounced fall for $G^{\prime}(\omega)$, leading to a crossover, which marks the change of behavior from solid-like to viscous-like. At very high strain amplitude $(\gamma>$ 
$600 \%)$, the viscous modulus dominated and the elastic component became negligible (less than $1 \%$ of $G$ ” $(\omega)$ ). Viscous behavior was recovered in the high strain amplitude regime only. Prior to it, blood plasma exhibited an elastic-like regime.

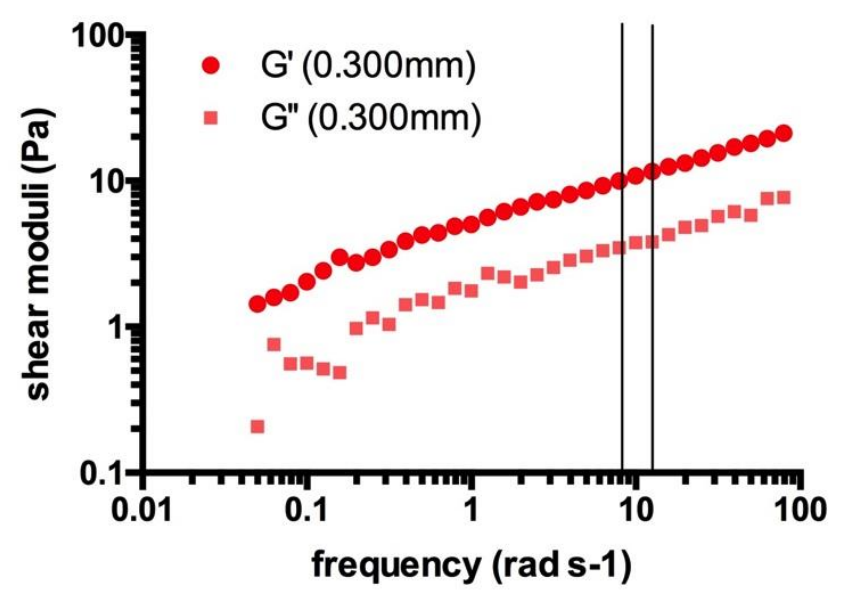

Figure 1. Measurements in well-controlled wetting (alumina surfaces): dynamic moduli $\left(G^{\prime}(\bullet)\right.$ and $\left.G^{\prime \prime}(\square)\right)$ of a $0.300 \mathrm{~mm}$ thick blood plasma versus frequency in low strain conditions (total wetting conditions - $1.5 \%$ strain amplitude, room temperature measurements). The vertical lines point out the heart rate domain $(1-1.5 \mathrm{~Hz})$. The error bar is about $5 \%$ of the measured values (logarithmic scale).

Similar features could be found in the thermal behavior (Figure 2). At low strain amplitude, the temperature was relatively homogeneous. As strain amplitudes increase, thermal bands of opposite temperatures appeared along the strain direction (flow regime $\gamma>200 \%$ ). The split of the fluid in opposite temperature variation consists of cold and hot regions aligned along the strain and a thermal compensation between the generated bands. The hot band is located close to the moving plate while the cold band is above the hot bands. The maximum temperature variation amplitude was reached at large strain (2500\%) reaching $|\Delta \mathrm{T}|=0.04 \pm 0.01^{\circ} \mathrm{C}$ (Figure 2). 


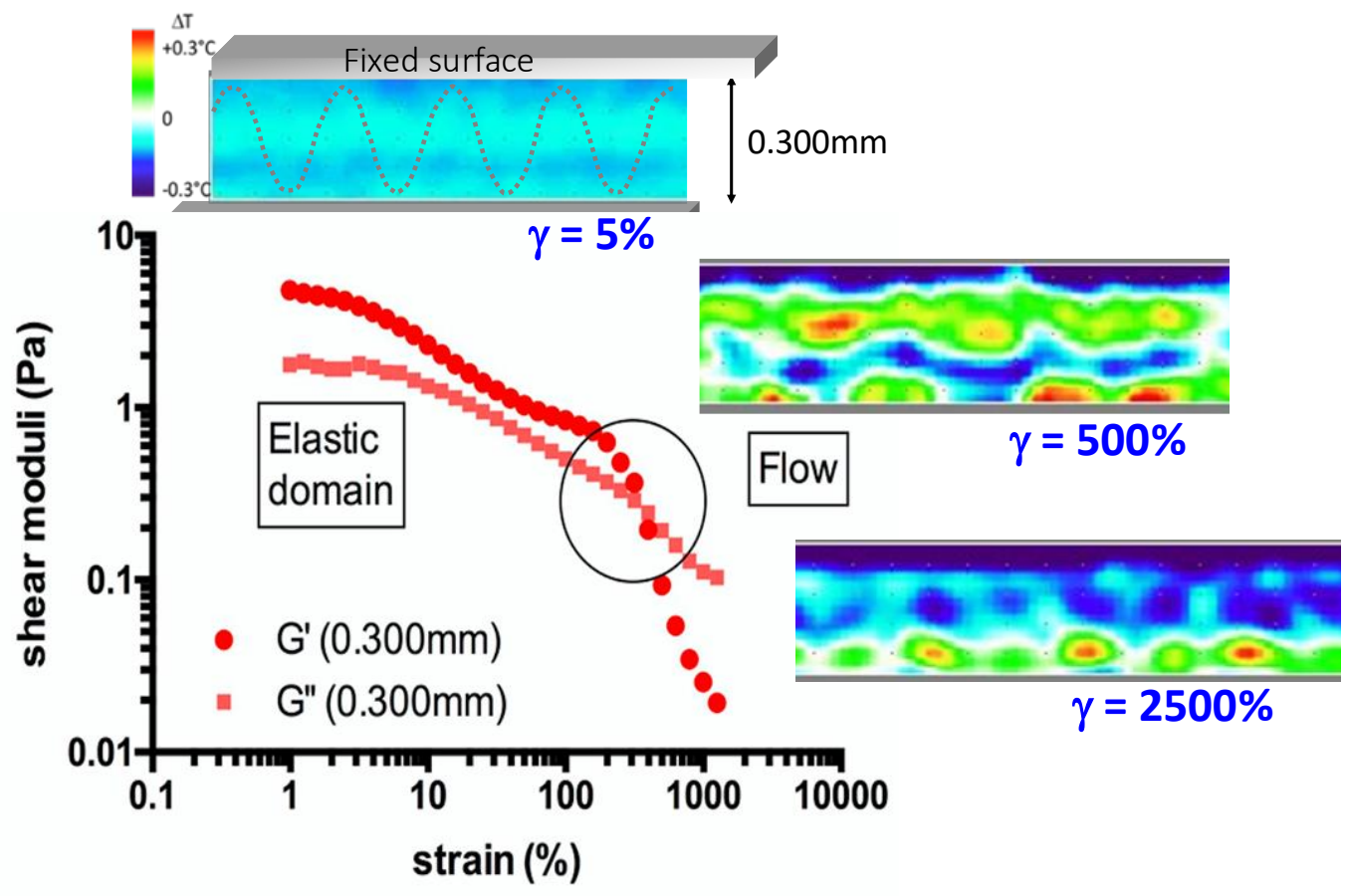

Figure 2. Human blood plasma exhibits, in the heart rate domain (here $\omega=10 \mathrm{rad} / \mathrm{s}$ ), an elastic-like response at low strain, the elastic modulus $G^{\prime}(\bullet)$ being larger than $G^{\prime \prime}(\square)$ for $\gamma<200 \%$. At larger deformation, a strain-induced transition from elastic to apparent viscous regime is observed and that is simultaneous with the emergence of contrasted thermal zones of sizeable amplitude $\pm 0.2^{\circ} \mathrm{C}$ (in situ microthermal cartography in the gap view). Measurements carried out at $0.300 \mathrm{~mm}$ gap thickness using total wetting conditions. Due to instrumental limitation, higher strain amplitudes were not probed ( $\gamma_{0}$ evolves from $1 \%$ to $1200 \%$ ). The error bar is about $5 \%$ of the measured values (logarithmic scale).

Since the lowest strain amplitudes correspond to less perturbing conditions, the elastic response is the fundamental response of blood plasma. No thermal response is measurable: the temperature is rather homogeneous. This is consistent with a conservative (elastic) mechanism. The shear elasticity becomes progressively replaced by a viscous response as shear strains rise. The fluid becomes thermally heterogeneous, whereas the viscoelastic measurement indicates a viscous regime only (G” > $>$ '). A viscous regime is incompatible with a thermal inhomogeneity. Consequently, the apparent viscous behavior is the non-linear product of the mechanical action on the shear elasticity converted in local thermodynamic states. Similar strain dependence schemes were reported in liquids and viscoelastic fluids (polymer melts, glass formers) by different authors ${ }^{5-9,11-14,19-20}$. Similar thermo-elastic effects were identified in simple fluids (glycerol, polypropylene glycol) confirming that the elasticity is likely a generic property ${ }^{15}$. 
Strain amplitude sweep tests carried out at several sample thicknesses show that the elastic response is accessible up to $1.100 \mathrm{~mm}$ using optimized wetting conditions, but decreases with increasing gap width (Figure 3). The shear elastic property is therefore lost at high strain amplitudes and large thicknesses; i.e. away from equilibrium conditions. Figure 3 thus summarizes the evolution of the shear elasticity of blood plasma as a function of its thickness. The insert of Figure 3 shows the $G^{\prime}$-values at near-linear response (elastic plateau at low strain amplitude). Like explained above, the elastic plateau developed at very low strains $(\gamma<5 \%)$ and decreased until both moduli dropped abruptly at about $300 \%$, at which strain the sample yielded (compare also with Figure 2). After yielding, the shear elastic moduli converged and became indistinguishable from values measured at other gap thicknesses.

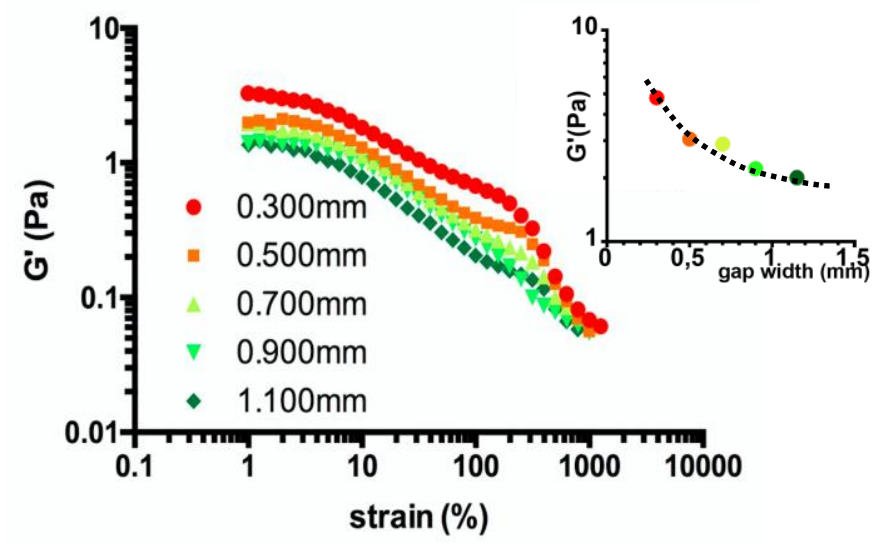

Figure 3. Influence of the scale (gap thickness) on the blood plasma shear elasticity measured at different thicknesses from $0.300 \mathrm{~mm}$ up to $1.100 \mathrm{~mm}$ versus strain amplitude (room temperature measurements, total wetting (alumina surfaces)): $\bullet: 0.300 \mathrm{~mm}, \square: 0.500 \mathrm{~mm}, \Delta$ : $0.700 \mathrm{~mm}, \nabla: 0.900 \mathrm{~mm}, \varangle: 1.150 \mathrm{~mm}$. The insert summarizes the evolution of the low strain amplitude shear elasticity (values near to the elastic plateau, logarithmic scale) versus gap thickness; measurements in high wetting conditions (alumina surfaces). The error bar is about $5 \%$ of the measured values (logarithmic scale).

\section{Discussion and Conclusion}

We have revealed that blood plasma exhibited a finite shear elastic response within a frequency range that covers the human heart rate if the interfacial contact is optimized. Its response to an oscillatory shear stress excitation in nearly static conditions (down to $0.04 \mathrm{rad} / \mathrm{s}$ upon applying low strain amplitude perturbation) is fundamentally elastic-like. Thus, for blood plasma to flow, the strain impulse has to overpass an elastic threshold. Significant shear strains and pulsations are needed to overcome the fundamental elastic property of blood plasma. Our results support recent findings, showing that blood plasma is viscoelastic when exposed to extensional deformation ${ }^{3,4}$. Additionally we have demonstrated the dimensional 
properties of shear elasticity by highlighting its reinforcement by the elastic threshold when the probed volume was reduced. Reciprocally, the elastic behavior is progressively replaced at large scale and/or at large strain amplitudes by a flow regime.

The present study evidences that a modification of the boundary surface has deep consequences for flow. Since the scale and the adhesion of blood plasma to the wall determine its strain-dependent behavior, the physico-chemistry of the interfacial boundary highly affects the volume flow through channels. An immobile plasma layer (not only at vessel walls) diminishes the available space for blood transport. It might even influence the material transport towards the wall and through it by selective binding. To optimize biomedical devices (e.g., extracorporeal membrane oxygenation, hemodialysis systems) and protocols (e.g., such as model blood flow through such devices), controlled interfacial conditions and true dimensional scales that reflect the planned application are key parameters. Since the threshold for flow is low, the boundary effect will be less significant at high flows.

In blood vessels, the role of plasma for the boundary surface is essential. The immersion of the endothelial glycocalix in plasma generates a stabile hydrogel layer (endothelial glycocalix layer, EGL) of several hundreds' nanometers in width that sieves molecules, hinders excessive fluid flux into the interstitium ${ }^{27-30}$, and limits the microvascular perfusion heterogeneity ${ }^{31}$. Therefore, flow does not start immediately at the endothelial cell bilayer but at a distance. Measurements of the flow resistance estimate this distance in the micrometer range from the endothelial cell ${ }^{32}$. The immobile part of the cellfree layer in vessels is therefore larger than the EGL width. The role of plasma is fundamental in this context because it contains the same main components as the glycocalix layer and can couple perfectly to it. Reitsma and co-authors described the surface layer as highly dynamic due to the synergetic interaction of soluble plasma components with the membrane bound components of the glycocalix ${ }^{33}$. The interplay between the two layers is disrupted when one part is degraded, which is the case at enzymatic treatment ${ }^{34}$.

The identification of the elastic behavior of blood plasma at low strain (Fig.1-3) reveals thus the dynamic range for which the EGL might be in full interaction with the plasma. The elasticity was highlighted by optimizing the conditions of the stress transfer that appear as playing a key role in the dynamic characterization of fluids ${ }^{5-9,14-15}$. "Static" shear elasticity reveals collective properties that involve long-range molecular like hydrogen bond interconnectivity. The thermal heterogeneity at large strain is also coherent with a thermo-mechanical coupling only possible through the deformation of a pre-existent shear elasticity as already observed in simple fluids and confirming that the elasticity is likely a generic fluidic property ${ }^{5-9},{ }^{15}$. Blood plasma definitively cannot be treated as a viscous fluid; this assumption strongly underestimates its properties and physiological relevance. 
Limitations: We propose an optimized system but since the walls are rigid, it can neither take into account the elasticity of the vessel wall (pulse pressure), nor the deformation of the glycocalix layer. Also, while the plasma is stabilized, long time scale experiments (hours) are however not possible, a crust being created on the fluid surface, especially at low gap widths. The thermal study is also limited by a poor spatial resolution $(20 \mu \mathrm{m})$ and by a limited dynamics. A high frequency recording of thermal images is hardly accessible but not necessary in the frame of physiological conditions.

\begin{tabular}{|l|c|}
\hline \multicolumn{1}{|c|}{ Parameter } & Tested range \\
\hline Frequency $\omega(\mathrm{rad} / \mathrm{s})$ & $0.05 \mathrm{rad} / \mathrm{s}$ to $100 \mathrm{rad} / \mathrm{s}$ \\
\hline Strain amplitude $\gamma(\%)$ & 1 to $2000 \%$ \\
\hline Gap thickness $e(\mathrm{~mm})$ & $300 \mu \mathrm{m}$ up to $1150 \mu \mathrm{m}$ \\
\hline Contact angle $(\theta)$ & $\begin{array}{c}\text { Total wetting } \theta \approx 0 \\
\text { (macroscopically) }\end{array}$ \\
\hline
\end{tabular}

Table 1

Table 1 indicates parameters varied for the study and the ranges of their use. These settings were chosen on basis of anatomical and physiological circumstances. The gap thickness of $300 \mu \mathrm{m}$ reflects the diameter of a large arteriole, whereas $1100 \mu \mathrm{m}$ reflects the diameter of a medium artery. In these resistance vessels the immobile part of the cell-free plasma layer is most relevant to blood flow. The physiologic heart rate covers 6 (at rest) and $19 \mathrm{rad} / \mathrm{s}$ (during exercise). This frequency range was included into the frequency sweep tests (Figure 1). Shear strain amplitudes differ across the vessel cross-section, being zero in the center of the flow, and maximal above the endothelial surface layer. Mean arterial wall shear rates are in the range of 300-500 s s, capillary wall shear rates are higher than $1000 \mathrm{~s}^{-1} 35$. Thus, the strain amplitude of $1 \%$ reflects conditions in the central part of the flow profile whereas the high strain amplitude (2000\%) reflect conditions in its parabolic part. It should also be taken into account that within the glycocalyx layer, to which blood plasma is attached, there may be deformation amplitudes of $1.5 \%{ }^{36}$.

Author's contributions: all authors contribute equally to this work.

Data availability: The data that support the findings of this study are available from the corresponding author upon reasonable request. 


\section{References:}

1. R. Fahraeus, and T. Lindqvist, The viscosity of the blood in narrow capillary tubes, Am. J. Physiol. 96 526-568 (1931)

2. T.H. Lijnema, J.R. Huizenga, J. Jager, A.J. Mackor, C.H. Gips, Gravimetric determination of the water concentration in whole blood, plasma and erythrocytes and correlations with hematological and clinicochemical parameters, Clin. Chim. Acta 214 129-138 (1993).

3. M. Brust, C. Schaefer, R. Doerr, L. Pan, M. Garcia, P.E. Arratia, C. Wagner. Rheology of human blood plasma: viscoelastic versus Newtonian behavior. Phys. Rev. Lett., 110 078305-5 (2013).

4. S. Varchanis, Y. Dimakopoulos, C. Wagner, J. Tsamopoulos. How viscoelastic is human blood plasma? Soft Matter 14, 4238-4251 (2018).

5. L. Noirez, H. Mendil-Jakani, and P. Baroni, The missing parameter in rheology: hidden solid-like correlations in viscous liquids, polymer melts and glass formers, Polym. Int. 58 962-968 (2009).

6. L. Noirez, and P. Baroni, Revealing the solid-like nature of glycerol at ambient temperature, J. Mol. Struct. 972 16$21(2010)$.

7. L. Noirez, and P. Baroni, Identification of a low-frequency elastic behaviour in liquid water, J. Phys. Condens. Matter 24372101 (2012).

8. L. Noirez, Importance of interfacial interactions to access shear elasticity of liquids and understand flow. Oil Gas Sci. Technol. Rev. I. F. P. Energ. Nouv. 72 10-17 (2017).

9. B. V. Derjaguin, U. V. Bazaron, Kh. D. Lamazhapova, and B. D. Tsidypov, Shear elasticity of low-viscosity liquids at low frequencies, Phys. Rev. A 422255 (1990).

10. J. Frenkel, Kinetic Theory of Liquids (Clarendon Press, 1946).

11. K. Trachenko, Lagrangian formulation and symmetrical description of liquid dynamics, Phys. Rev. E 96062134 (2017).

12. A. Zaccone, K. Trachenko, Explaining the low-frequency shear elasticity of confined liquids, PNAS $11719653-$ 19655 (2020).

13. Phillips, A.E.; Baggioli, M.; Sirk, T.W.; Trachenko, K.; Zaccone, A. Universal L ${ }^{-3}$ finite-size effects in the viscoelastic-ity of amorphous systems. Phys. Rev. Mater., 5, 035602 (2021). 
14. Zaccone, A.; Noirez, L. Universal $\mathrm{G}^{\prime} \sim \mathrm{L}^{-3}$ law for the low-frequency shear modulus of confined liquids. J. Phys. Chem. Lett., 12, 650-657 (2021).

15. E. Kume, P. Baroni, L. Noirez, Strain-induced violation of temperature uniformity in mesoscale liquids, Scientific Reports, 1013340 (2020).

16. S. Weinbaum, J. M. Tarbell, and E. R. Damiano, The structure and function of the endothelial glycocalyx layer, Annual Rev. Biomed. Eng. 9 121-167 (2007).

17. J. M. Tarbell, and Z. D. Shi, Effect of the glycocalyx layer on transmission of interstitial flow shear stress to embedded cells, Biomech. Model Mechanobiol. 12 111-121 (2013).

18. T. D. Blake, Slip between a liquid and a solid: D.M. Tolstoi’s (1952) theory reconsidered, Colloids Surf. 47 135-145 (1990).

19. A. Bund, and G. Schwitzgebel, Viscoelastic properties of low-viscosity liquids studied with thickness-shear mode resonators, Anal. Chem. 70 2584-2588 (1998).

20. P. Lv, Z. Yang, Z. Hua, M. Li, M. Lin, and X. Dong, Measurement of viscosity of liquid in micro-crevice, Flow Measurement and Instrumentation 46 72-79 (2015).

21. G.R. Lazaro, A. Hernandez-Machado, I. Pagonabarraga, Rheology of red blood cells under flow in highly confined microchannels, Soft Matter 107195 (2014).

22. F. de Paula Santosa, E. de Camposa, M. Costac, F. C. Lourenço Melob, R. Yzumi Hondac, R. Pinto Motac. Superficial Modifications in TiO2 and A12O3 Ceramics, Materials Research, Vol. 6, 353-367 (2003).

23. T. Schmatko, H. Hervet, and L. Leger. Friction and Slip at Simple Fluid-Solid Interfaces: The Roles of the Molecular Shape and the Solid-Liquid Interaction, Phys. Rev. Lett. 94, 244501 (2005).

24. Viscoelastic Properties of Polymers, 3rd Edition, J. D. Ferry, John Wiley \& Sons Inc (1980).

25. Th. G. Mayerhöfer, S. Pahlow, J. Popp. Recent technological and scientific developments concerning the use of infrared spectroscopy for point-of-care applications, Spectrochimica Acta Part A: Molecular and Biomolecular Spectroscopy, 251, $119411(2021)$.

26. S. Sabbatini, C. Conti, G. Orilisi, E. Giorgini, Infrared spectroscopy as a new tool for studying single living cells: Is there a niche? Biomedical Spectroscopy and Imaging 6 (2017) 85-99 85, DOI 10.3233/BSI-170171.

27. R. Pries, T. W. Secomb, and P. Gaehtgens, The endothelial surface layer, Europ. J. Physiol. 440 653-666 (2000).

28. M. Gouverneur, B. van den Berg, M. Nieuwdorp, E. Stroes, and H. Vink, Vasculoprotective properties of the endothelial glycocalix: effects of fluid shear stress, J. Internal Medicine 259 393-400 (2006). 
29. H. Vink, and B. R. Duling, Identification of distinct luminal domains for macromolecules, erythrocytes, and leukocytes within mammalian capillaries, Circ. Res. 79 581-589 (1996).

30. L. Copley, Hemorheological aspects of the endothelium-plasma interface, Microvasc. Res. 8 192-212 (1974).

31. P. M. McClatchey, M. Schafer, K. S. Hunter, and J. E. Reusch. The endothelial glycocalyx promotes homogenous blood flow distribution within the microvasculature, Am. J. Phys. 311 H168-H176 (2016).

32. A.R. Pries, T.W. Secomb, H. Jacobs, M. Sperandio, K. Osterlohl, P. Gaehtgens P. Microvascular blood flow resistance: role of endothelial surface layer. Am J Physiol 273 H2272-2279 (1997).

33. S. Reitsma, D.W. Slaaf, H. Vink, M.A.M.J. van Zandvoort, M.G.A. oude Egbrink. The endothelial glycocalyx: composition, functions, and visualisation. Pflügers Archiv - Eur J Physiol 454 345-359 (2007). DOI: 10.1007/s00424-007$0212-8$

34. O. Yalcin, V.P. Jani, P.C. Johnson, P. Cabrales. Hemodynamics and the arteriolar red cell free layer of the rat cremaster muscle. Frontiers of Physiology 00168 (2018). DOI: 10.3389/fphys.2018.00168.

35. M.A. Panteleev, N. Korin, K.D. Reesink, D.L. Bark, J.M.E.M Cosemans, E.E. Gardiner, E. E., \& P.H. Mangin, Wall shear rates in human and mouse arteries: Standardization of hemodynamics for in vitro blood flow assays: Communication from the ISTH SSC subcommittee on biorheology. Journal of Thrombosis and Haemostasis, 19(2), 588-595, (2021) https://doi.org/10.1111/jth.15174

36. Y. Yao, PhD thesis, Massachusetts Institute of Technology, 2005 (https://dspace.mit.edu/handle/1721.1/35653). 